\title{
Assessment of Similarity Factor Using Different Weighting Approaches
}

\author{
Mukesh C. Gohel ${ }^{1,2}$, Krishnakant G. Sarvaiya ${ }^{2}$, \\ Neelima R. Mehta ${ }^{2}$, Chirag D. Soni ${ }^{2}$, Vinita U. Vyas ${ }^{2}$ \\ and Rikita K. Dave ${ }^{2}$
}

email:mukeshgohel@hotmail.com

\begin{abstract}
The objective of the present work was to determine the value of similarity factor $\left(f_{2}\right)$ using different values of the optional weight $(w)$ to consider the variability in dissolution data. Three approaches are proposed for establishing similarity of dissolution profiles using the Moore and Flanner equation. In the first approach, the optional weight $\left(\mathrm{w}\right.$ ) was calculated by taking the ratio of 50 to $\mathrm{f}_{2 \mathrm{Th}}$, where 50 was selected as it is the borderline value for similarity or dissimilarity of the batches and $\mathrm{f}_{2 \mathrm{Th}}$ is the conversion factor which takes into account variability between samples at each timepoint. In the second approach, the optional weight (w) was calculated by taking the ratio of the absolute difference of the percentage of drug dissolved between reference $(R)$ and test $(\mathrm{T})$ formulations to $10 \%$ of percentage of drug dissolved from the reference formulation at each timepoint to consider the variability between samples with more specificity. In the third approach, the weight was calculated from the equation (1+SD/ maximum allowed SD) where standard deviation (SD) was calculated from 144 data points of absolute difference between $R$ and $T$ of 12 reference and 12 test formulations. The maximum allowed SD was arbitrarily chosen as 10 to consider within-sample variability as well as variability between samples. The calculation steps for estimating $\mathrm{f}_{2}$ are explained using the literature reported values of dissolution study. The results of the proposed approaches are compared with the classical approach of calculating $f_{2}(w=1)$. In some cases, the status of similarity changed to dissimilarity when the proposed approaches were adopted. The use of all three approaches is recommended in borderline cases of similarity. On the basis of consideration of variability, the three approaches are given preference in the order of Approach 3> Approach 2> Approach 1. Approach 3 may arouse new interest in $\mathrm{f}_{2}$ among industrial pharmacists and regulatory personnel as the within-samples variability and between-samples variability in dissolution data are considered in calculation of $\mathrm{f}_{2}$.
\end{abstract}

\section{Introduction}

rof he dissolution test is used to achieve different goals during the life cycle of a drug. At the time of registration and beyond, it is used for quality control purposes. For the comparison of dissolution profiles, model-dependent and model-independent methods are reported. ${ }^{1}$ The $f_{2}$ metric is extensively used because the US FDA endorses it. In a number of recent guidance documents, the FDA has placed more emphasis on the meaningful comparison of dissolution profiles. For example, the FDA Scale-Up and Post Approval Changes Modified Release (SUPAC MR) guidance indicates that similar dissolution profiles for approved and modified formulations are acceptable justification for certain levels of change without prior FDA approval or the need to perform bioequivalence studies. ${ }^{2}$ US FDA's guidances for industry on dissolution testing of immediate release solid oral dosage formulations and bioavailability and bioequivalence study guidance for oral dosage forms recommend the use of a model-independent mathematical approach proposed by Moore and Flannerfor calculating dissimilarity factor $\left(f_{1}\right)$ and similarity factor $\left(f_{2}\right)$ across a suitable time interval..$^{3-5}$ FDA recommends the use of $\mathrm{f}_{2}$ value to compare the dissolution data when the coefficient of variation is not more than $20 \%$ at the earlier time point and not more than $10 \%$ at other dissolution time points. ${ }^{3}$ Dissolution tests should preferably be conducted using validated automatic equipment and a precise assay method when attempting to establish similarity between a reference and a test formulation.

Vertzoni et al. ${ }^{6}$ pointed out that when data sets have increased variability, the ability of $f_{2}$ to reflect the true difference is yet to be investigated. The variability in the dissolution data may be attributed to human errors, equipment related errors, or formulation-related factors. Bartoszynski et al.' pointed out that the procedure currently used by the FDA involves computing the mean amount dissolved at each time and then comparing the two mean curves. Bartoszynski further stated that this approach ignores all of the variability within sets of profiles, which from a statistical viewpoint is a serious limitation. The optional weight is generally
${ }^{1}$ Corresponding author
2 L. M. College of Pharmacy, P. O. Box 4011, Navrangpura,

Ahmedabad 380009 India 
taken as one $(w=1)$ while calculating $f_{2}$. Anderson et al. ${ }^{8}$ suggested that the weight could be adjusted to give high or low weighting to selected time points as required. The researchers stated that if it is important to achieve a certain dissolution level by $40 \mathrm{~min}$, the 40 -min time point should be given a high weighting. ${ }^{8}$ Adams et al. ${ }^{9}$ reported that the measurement at each time point can be weighted according to its importance in the dissolution curve using the optional weight $(w)$. The investigators also reported that the $\mathrm{f}_{2}$ does not take into account the within-batch variability or the correlation between data. ${ }^{9}$ Sarandasa et al. ${ }^{10}$

suggested that since the similarity factor $\left(\mathrm{f}_{2}\right)$ is a function of mean differences and does not take into account the differences in dissolution within the test and reference batches, a careful interpretation is warranted when $\mathrm{f}_{2}$ is used as a similarity factor, especially when the variance of the profile is very different.

The optional weight $(w)$ has not been thoroughly explored by pharmaceutical scientists; hence the present work has been undertaken.

\section{Method}

The equation proposed by Moore and Flanner ${ }^{5}$ is as follows:

$$
f_{2}=50 \log \left\{\left[1+\frac{1}{n} \sum_{t=1}^{n} w_{t}\left(R_{t}-T_{t}\right)^{2}\right]^{-0.5} \times 100\right\}
$$

where $f_{2}$ is similarity factor, $n$ is the number of observations, $w_{t}$ is optional weight, $R_{t}$ is percentage drug dissolved from reference formulation, and $T_{t}$ is percentage drug dissolved from test formulation. The dissolution data reported by Shah et al. ${ }^{11}$ were used to calculate $f_{2}$ in the present work (Table 1).

The three weighting approaches are presented in this work.

\section{Approach 1}

Conventionally, a test batch is considered similar to that of a reference batch if the $f_{2}$ value of the two profiles is in between 50 and 100. The lower acceptable value (i.e.,50) corresponds to $10 \%$ average absolute difference between a reference product and a test product at each timepoint. In reality, a dissolution study will show different values of difference between $\mathrm{R}$ and $\mathrm{T}$ at each timepoint. This variability will be referred to as within-sample variability in this study. It was arbitrarily decided to give weight equal to one if the absolute difference between a reference and a test product is 10 . Shah et al. ${ }^{11}$ have reported the theoretical values of similarity factor for specific percentage difference

Table 1. Dissolution data for calculating $f_{2}$ values $^{11}$

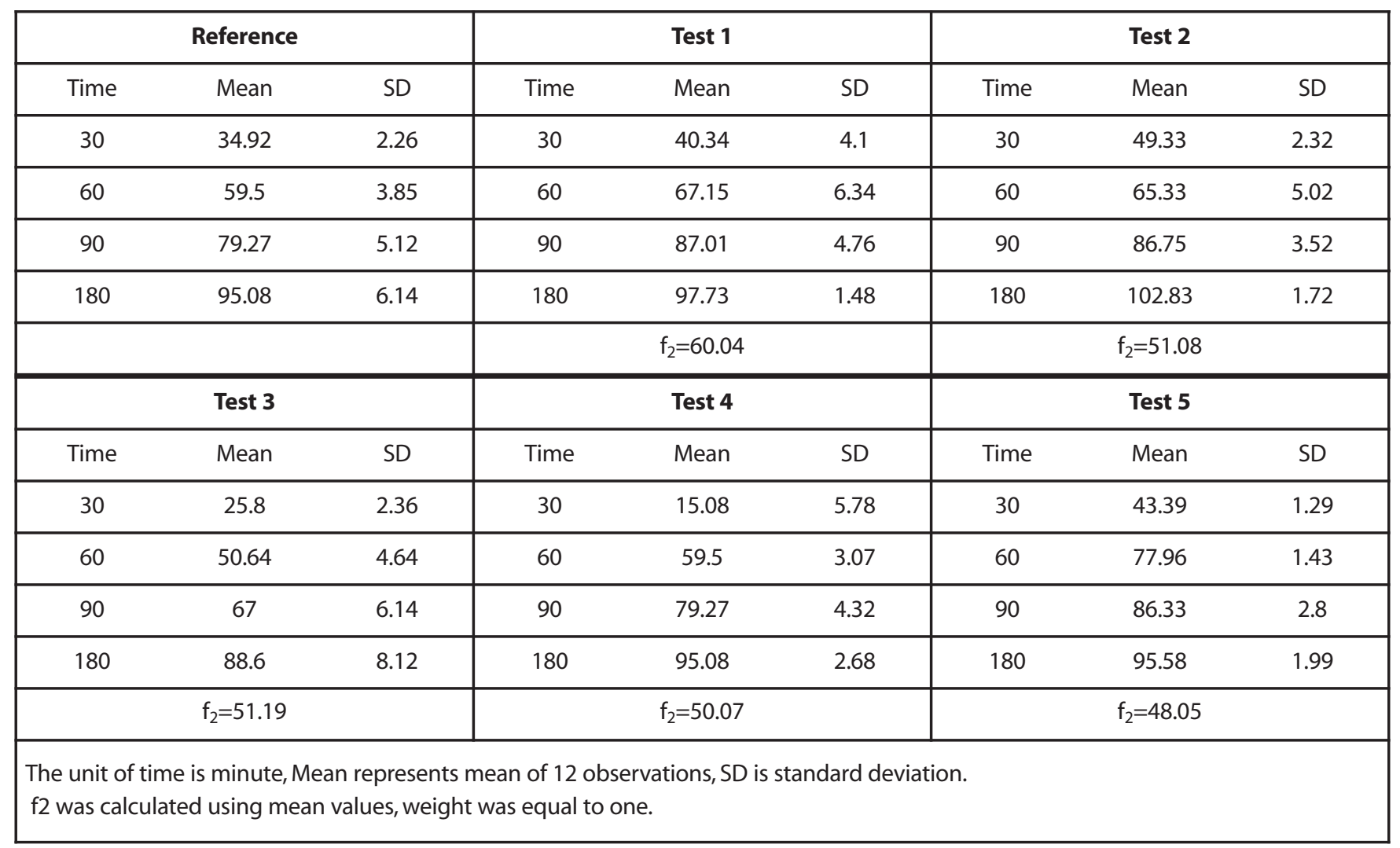


Table 2. Calculation of conversion factor ( $f 2 T h$ ) and weight (W) for different values of difference between R and T (Approach 1)

\begin{tabular}{|c|c|c|c|c|c|c|c|c|c|c|c|}
\hline R-T & $f_{2 T h}$ & $\mathbf{w}$ & R-T & $\mathbf{f}_{2 \mathrm{Th}}$ & $\mathbf{w}$ & $\mathbf{R}-\mathbf{T}$ & $f_{2 T h}$ & $\mathbf{w}$ & $\mathbf{R}-\mathbf{T}$ & $f_{2 T h}$ & $\mathbf{w}$ \\
\hline 0 & 100.00 & 0.50 & 5 & 64.63 & 0.77 & 10 & 49.89 & 1.00 & 15 & 41.15 & 1.22 \\
\hline 0.5 & 97.58 & 0.51 & 5.5 & 62.63 & 0.80 & 10.5 & 48.84 & 1.02 & 15.5 & 40.44 & 1.24 \\
\hline 1 & 92.47 & 0.54 & 6 & 60.79 & 0.82 & 11 & 47.84 & 1.05 & 16 & 39.75 & 1.26 \\
\hline 1.5 & 87.20 & 0.57 & 6 & 60.79 & 0.82 & 11.5 & 46.88 & 1.07 & 16.5 & 39.09 & 1.28 \\
\hline 2 & 82.53 & 0.61 & 7 & 57.53 & 0.87 & 12 & 45.97 & 1.09 & 17 & 38.44 & 1.30 \\
\hline 2.5 & 78.49 & 0.64 & 7.5 & 56.06 & 0.89 & 12.5 & 45.09 & 1.11 & 17.5 & 37.81 & 1.32 \\
\hline 3 & 75.00 & 0.67 & 8 & 54.68 & 0.91 & 13 & 44.24 & 1.13 & 18 & 37.20 & 1.34 \\
\hline 3.5 & 71.94 & 0.69 & 8.5 & 53.38 & 0.94 & 13.5 & 43.42 & 1.15 & 18.5 & 36.61 & 1.37 \\
\hline 4 & 69.24 & 0.72 & 9 & 52.15 & 0.96 & 14 & 42.64 & 1.17 & 19 & 36.03 & 1.39 \\
\hline 4.5 & 66.82 & 0.75 & 9.5 & 50.99 & 0.98 & 14.5 & 41.88 & 1.19 & 19.5 & 35.47 & 1.41 \\
\hline
\end{tabular}

between a reference and a test product (2\%-83,5\%-65, $10 \%-$ $50,15 \%-41$, and 20\%-36).

In this approach, at each timepoint, the optional weight (w) was calculated by taking the ratio of 50 to $f_{2 T h}$, where 50 is selected as the borderline value of similarity or dissimilarity of the batch as per acceptance criteria of the similarity factor $\left(f_{2}\right)$, and $f_{2 T h}$ is the conversion factor that takes into account variability between samples at each timepoint. The theoretical value of $f_{2 T h}$ at each timepoint was calculated using the seminal equation suggested by Moore and Flanner. ${ }^{5}$ The conversion factor $\left(f_{2 \mathrm{Th}}\right)$ was calculated by the following equation:

$$
f_{2 T h}=50 \times \log \left\{\left[1+(R-T)^{2}\right]^{-0.5} \times 100\right\}
$$

Table 2 depicts the $f_{2 T h}$ and $w\left(=50 / f_{2 T h}\right)$ values for different values of R-T. For example, weight with 5\% observed average absolute difference between reference and test is equal to $50 / 65(=0.77)$. Similarly weight was calculated for a range of 0 to $19.5 \%$ difference between reference and test formulations. For the calculation of modified similarity factor $\left(f_{2-m 1}\right)$, the literature reported values of mean dissolution data of a reference batch and five test batches were used. ${ }^{11}$ The results are summarized in Table 3.

\section{Approach 2}

In our earlier publication, we proposed an alternative method to calculate $\mathrm{f}_{2 .}{ }^{12}$ In place of 10 as the absolute percentage difference between $\mathrm{R}$ and $\mathrm{T}$, we proposed the use of $10 \%$ of percentage of drug dissolved from reference product as allowable deviation in the dissolution results of a test product. To consider variability between samples with more specificity, the optional weight (w) was calculated by taking the ratio of the absolute difference of mean percentage drug dissolved between $\mathrm{R}$ and $\mathrm{T}$ to $10 \%$ of percentage of drug dissolved from the reference formulation at each timepoint. The calculation for weight by this approach is shown in Table 4.

As shown in Table 4, 10\% of percentage of drug dissolved from reference formulation is equal to $3.492 \%$ at $30 \mathrm{~min}$. Therefore, according to this approach when the difference of percentage of drug dissolved between $\mathrm{R}$ and $\mathrm{T}$ is $3.492 \%$, the optional weight will be equal to one. Weight less than one was assigned to values lower than $10 \%$ of percentage of

Table 3. Values of similarity factor (f2-m1) for different test batches (Approach 1)

\begin{tabular}{|c|c|c|c|c|c|c|}
\hline \multicolumn{7}{|c|}{ Sample calculation for reference and test 1} \\
\hline Time & $\mathbf{R}$ & $\mathbf{T}$ & $\mathbf{R}-\mathbf{T}$ & $(\mathrm{R}-\mathrm{T})^{2}$ & $\mathbf{w}$ & $w^{*}(R-T)^{2}$ \\
\hline 30 & 34.92 & 40.34 & 5.42 & 29.38 & 0.77 & 22.62 \\
\hline 60 & 59.50 & 67.15 & 7.65 & 58.52 & 0.91 & 53.49 \\
\hline 90 & 79.27 & 87.01 & 7.74 & 59.91 & 0.91 & 54.76 \\
\hline 180 & 95.08 & 97.73 & 2.65 & 7.02 & 0.66 & 4.63 \\
\hline \multicolumn{7}{|c|}{ Similarity factors } \\
\hline ( $R$ and $T 1)$ & \multicolumn{2}{|c|}{$f_{2-m 1}=61.44$} & \multicolumn{2}{|c|}{$f_{2}=60.04$} & & \\
\hline ( $R$ and $T 2)$ & \multicolumn{2}{|c|}{$f_{2-m 1}=50.60$} & \multicolumn{2}{|c|}{$f_{2}=51.08$} & & \\
\hline (R and T3) & \multicolumn{2}{|c|}{$f_{2}=48.05$} & \multicolumn{2}{|c|}{$f_{2}=51.19$} & & \\
\hline (R and T4) & \multicolumn{2}{|c|}{$f_{2-m 1}=46.21$} & \multicolumn{2}{|c|}{$f_{2}=50.07$} & & \\
\hline ( $R$ and T5) & \multicolumn{2}{|c|}{$f_{2-m 1}=46.14$} & \multicolumn{2}{|c|}{$f_{2}=48.05$} & & \\
\hline \multicolumn{7}{|c|}{$\begin{array}{l}\mathrm{W}=50 / \mathrm{f}_{2 T h} \text { where, } \mathrm{f}_{2 T h} \text { :Conversion factor. } \\
\mathrm{f}_{2-\mathrm{m} 1}: \text { Similarity factor calculated using approach } 1 \\
\mathrm{f}_{2}: \text { Similarity factor calculated using conventional method }\end{array}$} \\
\hline
\end{tabular}


drug dissolved from reference at each timepoint. The obtained values of modified similarity factor $\left(f_{2-m_{2}}\right)$ for the proposed and the classical method are shown in Table 4.

\section{Approach 3}

Costa ${ }^{13}$ proposed an alternative method to calculate similarity factor using individual values of dissolution results of reference and test formulations in place of average dissolution data of a reference and a test product. The method proposed by $\operatorname{Costa}^{13}$ was used to generate different values of absolute difference between reference and test formulations $\left[\left(R_{1}-T_{1}\right),\left(R_{1}-T_{2}\right), \ldots\left(R_{1}-T_{12}\right), \ldots\left(R_{12}-T_{12}\right)\right]$ at each sampling time in the dissolution test. The results presented by Shah et al. ${ }^{11}$ for reference product ( 12 units) and test product (12 units) were used to generate 144 values of absolute difference between a reference and a test formulation at the four sampling time points $(30,60,90,180 \mathrm{~min})$. The twelve units of test formulation will show different dissolution profiles and this variability is referred to as between samples variability in this study. The next step involved calculation of standard deviation (SD) of absolute difference between $R$ and Tat each sampling time point.

If the analyst-related variability and equipment-related variability is assumed as negligible, variability in dissolution data is not anticipated from ideally prepared reference or test formulation. Under these circumstances, the SD of absolute difference between $\mathrm{R}$ and $\mathrm{T}$ shall be zero. It was arbitrarily decided to give weight equal to one when standard deviation is equal to zero. Further, it is proposed that the value of weight should proportionately increase as SD increases. In this approach, the weight was calculated from

Table 4. Values of similarity factor (f2-m2) for different test batches (Approach 2)

\begin{tabular}{|c|c|c|c|c|c|c|}
\hline \multicolumn{7}{|c|}{ Sample calculation for reference and test 1} \\
\hline Time & $\mathbf{R}$ & $\mathbf{T}$ & $\mathbf{R}-\mathbf{T}$ & $(R-T)^{2}$ & $\mathbf{w}$ & $\mathbf{w}^{*}(\mathbf{R}-\mathrm{T})^{2}$ \\
\hline 30 & 34.92 & 40.34 & 5.42 & 29.38 & $1.55^{*}$ & 45.60 \\
\hline 60 & 59.50 & 67.15 & 7.65 & 58.52 & 1.29 & 75.24 \\
\hline 90 & 79.27 & 87.01 & 7.74 & 59.91 & 0.98 & 58.49 \\
\hline 180 & 95.08 & 97.73 & 2.65 & 7.02 & 0.28 & 1.96 \\
\hline \multicolumn{7}{|c|}{ Similarity factors } \\
\hline (R and T1) & \multicolumn{2}{|c|}{$\mathrm{f}_{2-\mathrm{m} 2}=58.36$} & \multicolumn{2}{|c|}{$f_{2}=60.04$} & & \\
\hline (R and T2) & \multicolumn{2}{|c|}{$\mathrm{f}_{2-\mathrm{m} 2}=40.10$} & \multicolumn{2}{|c|}{$f_{2}=51.08$} & & \\
\hline (R and T3) & \multicolumn{2}{|c|}{$f_{2-m 2}=45.60$} & \multicolumn{2}{|c|}{$f_{2}=51.19$} & & \\
\hline (R and T4) & \multicolumn{2}{|c|}{$f_{2-m 2}=31.29$} & \multicolumn{2}{|c|}{$f_{2}=50.07$} & & \\
\hline (R and T5) & \multicolumn{2}{|c|}{$f_{2-m 2}=37.37$} & \multicolumn{2}{|c|}{$f_{2}=48.05$} & & \\
\hline \multicolumn{7}{|c|}{$\begin{array}{l}\mathrm{W}=\mathrm{R}-\mathrm{T} / 10 \% \text { of } \mathrm{R}=5.42 / 3.492=1.55 \\
\mathrm{f}_{2-\mathrm{m} 2}: \text { Similarity factor calculated using approach } 2 \\
\mathrm{f}_{2}: \text { Similarity factor calculated using conventional method }\end{array}$} \\
\hline
\end{tabular}

the equation (1+SD/ maximum allowed SD). The maximum allowed SD was arbitrarily chosen as 10 to allow withinsamples as well as variability between samples. The weights, standard deviations and the values of similarity factor $\left(\mathrm{f}_{2-\mathrm{m} 3}\right)$ are shown in Table 5.

\section{Results and Discussions}

In all the proposed approaches, if the weight at each timepoint is equal to one, the $f_{2}$ will remain unchanged. Weights higher than one can cause a drop in value of $f_{2}$ and weights less than one can cause a rise in $\mathrm{f}_{2}$ value. In the first step, the similarity factor was calculated using classical technique by taking weight as one $(w=1)$. The reported values of dissolution data ${ }^{9}$ for reference and test formulations and the calculated values of $f_{2}$ are depicted in Table 1 . The dissolution profile of all the formulations except test 5 can be considered as similar since calculated $f_{2}$ is greater than 50 .

\section{Approach 1}

Table 3 displays the value of the similarity factor calculated by the conventional method $\left(\mathrm{f}_{2}\right)$ and by the methodology expressed under Approach 1 in the methods section $\left(f_{2^{-}-{ }_{1} 1}\right)$. In case of the conventional method, all batches except T5 and in case of the proposed method, all batches except $\mathrm{T} 4$ and $\mathrm{T} 5$ showed results in the acceptable range $\left(f_{2}>50\right)$. The dissimilarity of test formulation 4 was attributed to the consideration of weight on the basis of variability.

The result reveals that in borderline cases of similarity/dissimilarity ( $f_{2}$ is around 50 ); the investigator may be able to draw the correct conclusion by adopting this approach. The proposed approach may ensure more mean-

Table 5. Values of similarity factor (f2-m3) for formulation test 1 (Approach 3)

\begin{tabular}{|c|c|c|c|c|c|}
\hline \multicolumn{6}{|c|}{ Sample calculation for reference and test 1} \\
\hline Time & Mean(R-T) & S.D. & $\mathbf{w}$ & $(\mathrm{R}-\mathrm{T})^{2}$ & $\mathbf{w}^{*}(\mathbf{R}-\mathrm{T})^{2}$ \\
\hline 30 & 5.70 & 4.35 & 1.44 & 32.46 & 46.58 \\
\hline 60 & 7.81 & 6.75 & 1.68 & 61.04 & 102.23 \\
\hline 90 & 7.83 & 5.44 & 1.54 & 61.38 & 94.77 \\
\hline 180 & 3.12 & 2.52 & 1.25 & 9.71 & 12.16 \\
\hline \multicolumn{6}{|c|}{ Similarity factors } \\
\hline ( R and T1) & \multicolumn{2}{|c|}{$f_{2-m 3}=54.68$} & \multicolumn{2}{|c|}{$f_{2}=60.04$} & \\
\hline (R and T2) & \multicolumn{2}{|c|}{$f_{2-m 3}=46.88$} & \multicolumn{2}{|c|}{$f_{2}=51.08$} & \\
\hline (R and T3) & \multicolumn{2}{|c|}{$f_{2-m 3}=48.30$} & \multicolumn{2}{|c|}{$f_{2}=51.19$} & \\
\hline (R and T4) & \multicolumn{2}{|c|}{$f_{2-m 3}=46.46$} & \multicolumn{2}{|c|}{$f_{2}=50.07$} & \\
\hline (R and T5) & \multicolumn{2}{|c|}{$f_{2-m 3}=44.98$} & \multicolumn{2}{|c|}{$f_{2}=48.05$} & \\
\hline \multicolumn{6}{|c|}{$\begin{array}{l}\mathrm{w}=(1+\mathrm{SD} / \text { maximum allowed } \mathrm{SD})=(1+4.35 / 10)=1.435 \\
\mathrm{f}_{2-\mathrm{m} 3}: \text { Similarity factor calculated using approach } 3 \\
\mathrm{f}_{2}: \text { Similarity factor calculated using conventional method }\end{array}$} \\
\hline
\end{tabular}


ingful comparison of the dissolution profiles than the classical method since it encompasses the difference between $R$ and $T$ at each timepoint in dissolution testing. The proposed approach can be applied to integer values as well as fractional values of the difference between the percentage of drug dissolved of $R$ and $T$ at each timepoint.

\section{Approach 2}

The reported values of dissolution data ${ }^{11}$ and the calculated values of similarity factor $\left(\mathrm{f}_{2}\right)$ by this approach $\left(\mathrm{f}_{2-\mathrm{m} 2}\right)$ are depicted in Table 4 . The result of test 1 formulation indicates that this status of similarity is not changed since $\mathrm{f}_{2}$ is well above 50. It appears that the approach will find application in cases where $\mathrm{f}_{2}$ is close to 50 . In the test batches 2,3 , and 4 , the conclusion of similarity between $R$ and $T$ changed to dissimilarity when approach 2 was adopted for calculation of similarity factor. Approach 2 seems to be more sensitive than Approach 1 since only one test formulation (T1) showed similarity as compared to three test formulations (T1,T2 and T3) in Approach 1. Scientists are warned to find outliers and to consider them at the time of taking decision of similarity/dissimilarity.

\section{Approach 3}

An effort is made in this approach to address the issue raised by Bartoszynski, Adams, ${ }^{9}$ and Sarandasa ${ }^{10}$. The major advantage of the proposed method is that each and every dissolution data value and not just the average dissolution data are used. The standard deviation of different values (144) of absolute difference between $R$ and $T$ was calculated at each timepoint. Whenever within-samples variability and variability between samples will be observed, the calculated value of $f_{2-m 3}$ by the proposed method will be lower than the similarity factor $\left(\mathrm{f}_{2}\right)$ calculated by the classical method $(w=1)$. A large difference between two similarity factors $\left(f_{2}\right.$ and $\left.\mathrm{f}_{2-\mathrm{m} 3}\right)$ indicates high variability in the dissolution results. One should be careful when the status of similarity $\left(f_{2}>50\right)$ changes to the status of dissimilarity $\left(\mathrm{f}_{2-\mathrm{m}_{3}}<50\right)$.

The reported values of dissolution data ${ }^{11}$ of the reference and the five test formulations were used for calculation of similarity factor. Table 5 shows the values of the similarity factor by the proposed approach and the classical method. The status of similarity was changed from acceptable $\left(f_{2}>50\right)$ to unacceptable $\left(f_{2}<50\right)$ for test formulations $T 2, T 3$, and T4 . Approach 3 will always cause depression in the value of $f_{2 \text {; }}$ however, the depression $\left(f_{2}-f_{2-m}\right)$ will be proportional to variability. If the value of SD is high at all timepoints, a more significant depression is expected. If SD is zero at each timepoint, $\mathrm{f}_{2}$ will be equal to $\mathrm{f}_{2-\mathrm{m} 3}$.

The preliminary idea of variability can be obtained by calculating standard deviation of $R$ and $T$ at each timepoint. In critical cases of selecting a test formulation for bioequivalence study based on dissolution data, the use of this approach is recommended. The major disadvantage of the proposed approach is cumbersome calculation steps. Software can be developed to handle this problem. In borderline cases of similarity identified while granting exemption of detailed study for SUPAC MR products, the FDA may ask the manufacturer to submit data according to this approach.

\section{Conclusion}

The widely accepted method of calculating the similarity factor for comparison of dissolution profiles ignores the very important issue of variability in dissolution data. A careful introduction of weight based on variability may provide a meaningful resolution of this issue. The proposed approaches of considering weight add an additional dimension to the use of weight and evoke new interest in the area of comparison of dissolution profiles. Among the proposed approaches, the preferences is given in the order of Approach3> Approach2> Approach1. Approach 3 is given maximum preference, as it considers batch-to-batch as well as within-samples variability. Approach 2 is given more preference over Approach 1 because it considers batch-tobatch variability with more specificity as compared to Approach 1. For all the proposed approaches, when $R=T, f_{2}$ will be 100 and a value close to 100 indicates a superior formulation. The formulation and development scientist may use these approaches in borderline cases of similarity and for selection of superior test formulation for bioequivalence study, IVIVC, and for getting biowaivers on more scientific basis. The scientific community will be tempted to find other methods to calculate and use weight after reading this manuscript.

\section{References}

1. Polli, J. E.; Rekhi, G. S.; Augsburger, L. L.; Shah, V. P. Methods to compare dissolution profiles and a rationale for wide dissolution specification for metoprolol tartrate tablets. J.Pharm.Sci. 1997, 86(6), 690-700.

2. Guidance for Industry: SUPAC-MR Modified Release Solid Oral Dosage Forms, Scale-Up and Post-Approval Changes: Chemistry, Manufacturing and Controls, In Vitro Dissolution Testing and In Vivo Bioequivalence Documentation. US Food and Drug Administration, Rockville, MD, USA, 1997.

3. Guidance for Industry: Dissolution Testing of Immediate Release Solid Oral Dosage Forms. US Food and Drug Administration, Rockville, MD, USA, 1997.

4. Guidance for Industry: Immediate Release Solid oral Dosage Forms, Scale-Up and Post-Approval Changes: Chemistry, Manufacturing and Controls, In Vitro Dissolution Testing, and In Vivo Bioequivalence Documentation. US Food and Drug Administration, Rockville, MD, USA, 1995. 
5. Moore, J. W.; Flanner, H. H. Mathematical comparison of curves with an emphasis on in-vitro dissolution profiles. Pharm. Tech. 1996, 20(6), 64-74.

6. Vertzoni, M.; Symillides, M.; Iliadis, A.; Nicolaides, E.; Reppas, C. Comparison of simulated cumulative drug versus time data sets with indices. Euro. J. Pharm. Biopharm. 2003, 56, 421-428.

7. Bartoszynski, R.; Powers, J. D.; Herderick, E. E.; Pultz, J. A. Statistical comparison of dissolution curves. Pharmcol. Res. 2001, 43(4), 371-387.

8. Anderson, N. H.; Bauer, M.; Boussac, N.; Malek, R. K.; Munden, P. An evaluation of fit factors and dissolution efficiency for the comparison of in-vitro dissolution profiles. J. Pharm. Biomed. Anal. 1998, 17, 811-822.

9. Adams, E.; Maesschalck, R. D.; Spiegeleer, B. D.; Heyden, Y. V.; Verbeke, J. S.; Massart, D. L. Evaluation of dissolu- tion profiles using principal component analysis. Int. J. Pharm. 2001, 212, 42-53.

10. Sarandasa, H.; Krishnamoorthy, K. A multivariate tests for similarity of two dissolution profiles. J. Biopharm. Stat. 2005, 15, 265-278.

11. Shah,V. P.; Tsong,Y.; Sathe, P.; Liu, J. P. In vitro Dissolution Profile Comparison-Statistics and Analysis of the Similarity Factor, f. Pharm. Res. 1998, 15(6), 889-895.

12. Gohel, M. C.; Panchal, M. K. Refinement of Lower Acceptance Value of the Similarity Factor $\mathrm{f}_{2}$ in Comparison of Dissolution Profile. Dissolution Technologies 2002, 18-22.

13. Costa, P. An alternative method to evaluation of similarity factor in dissolution testing. Int. J. Pharm. 2001, $220,77-83$. 